TRANSACTIONS OF THE

AMERICAN MATHEMATICAL SOCIETY

Volume 361, Number 4, April 2009, Pages 1731-1747

S 0002-9947(08)04574-1

Article electronically published on October 31, 2008

\title{
PROPAGATION OF GEVREY REGULARITY FOR SOLUTIONS OF THE BOLTZMANN EQUATION FOR MAXWELLIAN MOLECULES
}

\author{
LAURENT DESVILLETTES, GIULIA FURIOLI, AND ELIDE TERRANEO
}

\begin{abstract}
We prove that Gevrey regularity is propagated by the Boltzmann equation with Maxwellian molecules, with or without angular cut-off. The proof relies on the Wild expansion of the solution to the equation and on the characterization of Gevrey regularity by the Fourier transform.
\end{abstract}

\section{INTRODUCTION}

This paper deals with a propagation property for the solution of the following Cauchy problem for the spatially homogeneous Boltzmann equation for Maxwellian molecules

$$
\left\{\begin{array}{l}
\partial_{t} f(v, t)=Q(f, f)(v, t), \\
f(v, 0)=f_{0}(v)
\end{array}\right.
$$

Here, $f(v, t): \mathbb{R}^{3} \times \mathbb{R}^{+} \longrightarrow \mathbb{R}$ is the probability density of a gas which depends only on the velocity $v \in \mathbb{R}^{3}$ at the time $t \geq 0$ and $Q$ is the quadratic Boltzmann collision operator in the case of Maxwellian molecules:

$Q(f, f)(v, t)=\int_{w \in \mathbb{R}^{3}} \int_{n \in S^{2}}\left(f\left(v_{*}, t\right) f\left(w_{*}, t\right)-f(v, t) f(w, t)\right) b\left(\frac{v-w}{|v-w|} \cdot n\right) \mathrm{d} n \mathrm{~d} w$.

Due to the physical assumptions that the gas evolves through binary, elastic collisions which are localized both in space and time, the relations between the velocities $\left(v_{*}, w_{*}\right)$ of two particles before the collision and $(v, w)$ after it are the following:

$$
\left\{\begin{array}{l}
v_{*}=\frac{v+w}{2}+\frac{|v-w|}{2} n, \\
w_{*}=\frac{v+w}{2}-\frac{|v-w|}{2} n,
\end{array}\right.
$$

where $n$ is a vector in $S^{2}$, the unit sphere in $\mathbb{R}^{3}$, and parametrizes all the possible pre-collisional velocities.

The collision kernel $b$, which is supposed to be nonnegative, is the function which selects in which way the pre-collisional velocities contribute to produce particles with velocity $v$ after the collision and is supposed (this is precisely the assumption

Received by the editors November 20, 2006.

2000 Mathematics Subject Classification. Primary 76P05; Secondary 35B65.

Key words and phrases. Homogeneous Boltzmann equation, cut-off and non-cut-off, propagation of regularity, Gevrey class.

(C)2008 American Mathematical Society Reverts to public domain 28 years from publication 
of Maxwellian molecules) to depend only on the cosine of the deviation angle $\theta$, namely

$$
\cos \theta=\frac{v-w}{|v-w|} \cdot n
$$

Finally, we will make the so-called non-cut-off assumption, which means that $b \notin$ $L_{\text {loc }}^{1}([-1,1])$ and, more precisely, we shall consider

$$
b(\cos \theta) \sim \frac{1}{(1-\cos \theta)^{\frac{5}{4}}}, \quad \theta \rightarrow 0 .
$$

From a physical point of view, that means that the gas molecules repel each other with a force inversely proportional to the fifth power of their distance and a great contribution to the integral collision term is given by the grazing collisions $(\theta \sim 0)$. From a mathematical point of view, the more general assumption

$$
b(\cos \theta) \sim \frac{1}{(1-\cos \theta)^{\gamma}}, \quad \theta \rightarrow 0, \quad 1<\gamma<2,
$$

will be considered in this work.

The assumption that the collision kernel $b$ is instead integrable on $[-1,1]$ is called a cut-off assumption. For more information about the Boltzmann equation and its physical meaning, the reader can consult, for instance, the review article by Villani [15].

Due to the singularity of the collision kernel at the origin, the integral term (1.1) is not meaningful if $f$ is not smooth, and so it is convenient to consider the weak form of the Boltzmann equation: for $\varphi \in C_{c}^{\infty}\left(\mathbb{R}^{3}\right)$,

$$
\begin{aligned}
& \frac{\mathrm{d}}{\mathrm{d} t} \int_{v \in \mathbb{R}^{3}} f(v, t) \varphi(v) \mathrm{d} v \\
& =\int_{v \in \mathbb{R}^{3}} Q(f, f)(v, t) \varphi(v) \mathrm{d} v \\
& =\int_{v \in \mathbb{R}^{3}} \int_{w \in \mathbb{R}^{3}} \int_{n \in S^{2}} f(v, t) f(w, t)\left(\varphi\left(v_{*}\right)-\varphi(v)\right) b\left(\frac{v-w}{|v-w|} \cdot n\right) \mathrm{d} n \mathrm{~d} w \mathrm{~d} v
\end{aligned}
$$

or even, with another point of simplification, in the Fourier variable,

$$
\left.\partial_{t} \hat{f}(\xi, t)=\int_{n \in S^{2}}\left(\hat{f}\left(\xi^{+}, t\right) \hat{f}\left(\xi^{-}, t\right)-\hat{f}(\xi, t) \hat{f}(0, t)\right) b\left(\frac{\xi}{|\xi|} \cdot n\right) \mathrm{d} n=\widehat{Q(f, f}\right)(\xi, t),
$$

as was firstly done by Bobylëv (see for instance [3]). Here we have used the standard notation

$$
\xi^{+}=\frac{\xi}{2}+\frac{|\xi|}{2} n, \quad \xi^{-}=\frac{\xi}{2}-\frac{|\xi|}{2} n
$$

The first results concerning the non-cut-off case for the weak equation go back to Arkeryd [2, in a more general setting. In [11, A. Pulvirenti and G. Toscani reformulated the existence theory starting from the equation in the Fourier variable both for the cut-off and non-cut-off cases. We briefly recall their method and their result, because they will be useful in the following. The classical approach is to find a solution of equation (1.3) through a limiting process on the solutions of a sequence of cut-off approximating problems in the following way. Let us consider the following sequence of bounded functions obtained by cutting out the singularity of $b$ at the origin:

$$
\bar{b}_{l}=\min (b, l), \quad l \in \mathbb{N},
$$


and let

$$
b_{l}^{*}=\int_{n \in S^{2}} \bar{b}_{l}\left(\frac{\xi}{|\xi|} \cdot n\right) \mathrm{d} n
$$

Then, define

$$
\beta_{l}=\frac{\bar{b}_{l}}{b_{l}^{*}}
$$

so that

$$
\int_{n \in S^{2}} \beta_{l}\left(\frac{\xi}{|\xi|} \cdot n\right) \mathrm{d} n=1
$$

and then consider the sequence of Cauchy problems

$$
\left\{\begin{array}{l}
\partial_{\tau} \varphi_{l}(\xi, \tau)=\int_{n \in S^{2}}\left(\varphi_{l}\left(\xi^{+}, \tau\right) \varphi_{l}\left(\xi^{-}, \tau\right)-\varphi_{l}(\xi, \tau) \varphi_{l}(0, \tau)\right) \beta_{l}\left(\frac{\xi}{|\xi|} \cdot n\right) \mathrm{d} n \\
\varphi_{l}(\xi, 0)=\hat{f}_{0}(\xi) .
\end{array}\right.
$$

A. Pulvirenti and G. Toscani proved first the existence and uniqueness of a solution $\varphi_{l}$ of the Cauchy problems (1.7). Then, letting

$$
\hat{f}_{l}(\xi, t):=\varphi_{l}\left(\xi, b_{l}^{*} t\right),
$$

they proved the convergence in a suitable setting of a subsequence of $\hat{f}_{l}$ to a solution of the Cauchy problem for the non-cut-off equation. More precisely, the result is as follows:

Theorem 1.1 (Pulvirenti, Toscani [1]). We consider an initial datum $f_{0} \geq 0$ satisfying the following assumptions:

$$
\begin{gathered}
\int_{\mathbb{R}^{3}} f_{0}(v) \mathrm{d} v=1, \quad \int_{\mathbb{R}^{3}} f_{0}(v) v_{i} \mathrm{~d} v=0, i=1,2,3, \quad \int_{\mathbb{R}^{3}} f_{0}(v)|v|^{2} \mathrm{~d} v=3, \\
\int_{\mathbb{R}^{3}} f_{0}(v)\left|\log f_{0}(v)\right| \mathrm{d} v<\infty,
\end{gathered}
$$

and the following Cauchy problem:

(1.8)

$$
\left\{\begin{array}{l}
\partial_{t} \hat{f}(\xi, t)=\int_{n \in S^{2}}\left(\hat{f}\left(\xi^{+}, t\right) \hat{f}\left(\xi^{-}, t\right)-\hat{f}(\xi, t) \hat{f}(0, t)\right) b\left(\frac{\xi}{|\xi|} \cdot n\right) \mathrm{d} n, \quad t>0 \\
\hat{f}(\xi, 0)=\hat{f}_{0}(\xi)
\end{array}\right.
$$

where $b$ is a nonnegative function of $L_{\mathrm{loc}}^{1}([-1,1[)$ satisfying (1.2).

Then, there exists a nonnegative solution $f \in C^{1}\left([0,+\infty), L^{1}\left(\mathbb{R}^{3}\right)\right)$ to (1.8) satisfying for all $t>0$ :

$$
\begin{gathered}
\int_{\mathbb{R}^{3}} f(v, t) \mathrm{d} v=1, \quad \int_{\mathbb{R}^{3}} f(v, t) v_{i} \mathrm{~d} v=0, i=1,2,3, \quad \int_{\mathbb{R}^{3}} f(v, t)|v|^{2} \mathrm{~d} v=3, \\
\int_{\mathbb{R}^{3}} f(v, t)|\log f(v, t)| \mathrm{d} v<\infty .
\end{gathered}
$$

Moreover, for all $t>0$, the Fourier transform $\hat{f}(\cdot, t)$ of the solution is obtained as the (uniform on compact sets) limit of a subsequence of the functions $\varphi_{l}\left(\cdot, b_{l}^{*} t\right) \in$ 
$C^{1}\left([0,+\infty), C_{b}\left(\mathbb{R}^{3}\right)\right)$, solutions of the cut-off Cauchy problems (1.7), which have the following explicit representation (called Wild's expansion):

$$
\varphi_{l}(\xi, \tau)=\mathrm{e}^{-\tau} \sum_{k=0}^{\infty} \varphi_{l}^{(k)}(\xi)\left(1-\mathrm{e}^{-\tau}\right)^{k}
$$

where

$$
\begin{aligned}
& \varphi_{l}^{(0)}(\xi)=\hat{f}_{0}(\xi), \\
& \varphi_{l}^{(k+1)}(\xi)=\frac{1}{k+1} \sum_{j=0}^{k} \int_{n \in S^{2}} \varphi_{l}^{(j)}\left(\xi^{+}\right) \varphi_{l}^{(k-j)}\left(\xi^{-}\right) \beta_{l}\left(\frac{\xi}{|\xi|} \cdot n\right) \mathrm{d} n .
\end{aligned}
$$

In [13, Toscani and Villani proved that under the hypotheses of Theorem 1.1, the solution for the non-cut-off equation is indeed unique, whereas the uniqueness of the solution for the cut-off problem (1.7) was already known.

This paper is devoted to a discussion of the following question: if the initial datum $f_{0}$ satisfies the upper bound

$$
\left|\hat{f}_{0}(\xi)\right| \leq K_{1} \mathrm{e}^{-K_{2}|\xi|^{s}}, \quad \xi \in \mathbb{R}^{3}, K_{1} \geq 1, K_{2}>0, s>0,
$$

does the solution (of the cut-off or non-cut-off Boltzmann equation with Maxwellian molecules) keep on satisfying the same property? The answer is positive, provided that $s \in] 0,2]$, and we allow the constants $K_{1}, K_{2}$ to be different from those of the initial datum.

More precisely, the result we are going to prove is the following:

Theorem 1.2. Let $b$ be a nonnegative function of $L_{\mathrm{loc}}^{1}([-1,1[)$ satisfying (1.2), and let $f_{0}$ be a nonnegative function satisfying

$$
\begin{gathered}
\int_{\mathbb{R}^{3}} f_{0}(v) \mathrm{d} v=1, \quad \int_{\mathbb{R}^{3}} f_{0}(v) v_{i} \mathrm{~d} v=0, i=1,2,3, \quad \int_{\mathbb{R}^{3}} f_{0}(v)|v|^{2} \mathrm{~d} v=3, \\
\int_{\mathbb{R}^{3}} f_{0}(v)\left|\log f_{0}(v)\right| \mathrm{d} v<\infty .
\end{gathered}
$$

If $f_{0}$ is such that

$$
\sup _{\xi \in \mathbb{R}^{3}}\left|\hat{f}_{0}(\xi)\right| \mathrm{e}^{K_{2} \psi\left(|\xi|^{2}\right)} \leq K_{1}
$$

for some $K_{1} \geq 1, K_{2}>0$ and for some function $\psi:[0,+\infty) \rightarrow[0,+\infty)$, such that $\psi(r) \leq r$ for $r$ large enough, $\psi(r) \rightarrow+\infty$ for $r \rightarrow+\infty$, and satisfying the condition:

$(H)$ there exists $\bar{R} \geq 1$ such that

$$
\psi\left(\lambda^{2}|\xi|^{2}\right) \geq \lambda^{2} \psi\left(|\xi|^{2}\right), \quad \text { when } \quad 0 \leq \lambda \leq 1, \quad \lambda|\xi| \geq \bar{R},
$$

then there exist $R_{0}>0, K>0$ such that the unique solution of the Cauchy problem (1.8) with $f_{0}$ as initial datum satisfies

$$
\begin{aligned}
& \sup _{|\xi|<R_{0}}|\hat{f}(\xi, t)| \mathrm{e}^{K|\xi|^{2}} \leq 1, \quad t \geq 0, \\
& \sup _{|\xi| \geq R_{0}}|\hat{f}(\xi, t)| \mathrm{e}^{K \psi\left(|\xi|^{2}\right)} \leq 1, \quad t \geq 0 .
\end{aligned}
$$


We would like to point out that condition $(H)$ is satisfied for every concave function $\psi:[0,+\infty) \rightarrow[0,+\infty)$, such that $\psi(0)=0$ such as, for instance, $\psi(t)=$ $|t|^{\mu}$ for $\mu \in(0,1)$ and also for functions like $\psi(t)=|t|^{\mu}|\log t|^{\nu}$ for $\mu \in(0,1), \nu>0$.

Denoting by $G^{\nu}\left(\mathbb{R}^{3}\right)$ the space of Gevrey functions and by $G_{0}^{\nu}\left(\mathbb{R}^{3}\right)$ the space of Gevrey functions with compact support (we shall recall in Section 4 their definition), we are able to deduce from the previous result the propagation along the solution of a Gevrey-type regularity satisfied by the initial datum.

Corollary 1.3. Let $b$ be a nonnegative function of $L_{\text {loc }}^{1}([-1,1[)$ satisfying (1.2), and let $f_{0}$ be a nonnegative function satisfying

$$
\begin{gathered}
\int_{\mathbb{R}^{3}} f_{0}(v) \mathrm{d} v=1, \quad \int_{\mathbb{R}^{3}} f_{0}(v) v_{i} \mathrm{~d} v=0, i=1,2,3, \quad \int_{\mathbb{R}^{3}} f_{0}(v)|v|^{2} \mathrm{~d} v=3, \\
\int_{\mathbb{R}^{3}} f_{0}(v)\left|\log f_{0}(v)\right| \mathrm{d} v<\infty .
\end{gathered}
$$

i) If $\nu>1$ and $f_{0} \in G_{0}^{\nu}\left(\mathbb{R}^{3}\right)$, then the solution $f(\cdot, t)$ of the Cauchy problem (1.8) is in $G^{\nu}\left(\mathbb{R}^{3}\right)$, uniformly for all $t \geq 0$.

ii) If $\nu \geq 1, f_{0} \in G^{\nu}\left(\mathbb{R}^{3}\right) \cap \mathcal{S}^{\prime}\left(\mathbb{R}^{3}\right)$, and moreover $\operatorname{satisfies}^{\sup _{\xi \in \mathbb{R}^{3}}}\left|\hat{f}_{0}(\xi)\right| \leq$ $K_{1} e^{-K_{2}|\xi|^{\frac{1}{\nu}}}$ for $K_{1} \geq 1, K_{2}>0$, then the solution $f(\cdot, t)$ of the Cauchy problem (1.8) is in $G^{\nu}\left(\mathbb{R}^{3}\right)$, uniformly for all $t \geq 0$.

The question whether an extra property satisfied by the initial datum $f_{0}$ propagates along the solution has already been addressed concerning Sobolev or Lebesgue regularity. In [7, Desvillettes and Mouhot proved the uniform propagation of $L^{p}$ moments for both the cut-off and non-cut-off equations (cf. also [8], 9], 10] for earlier works on the propagation of $L^{p}$ regularity). In 4, Carlen, Gabetta and Toscani proved for the cut-off equation that also all the $H^{s}$ Sobolev norms remain uniformly bounded if they exist initially (cf. also [10] for related results in the case of hard potentials and hard spheres). When the non-cut-off equation is considered, the same is true, and moreover the $H^{s}$ norms are immediately created (cf. [5, 6]). In [14, Ukai proved for both the cut-off and non-cut-off equations that a regularity property of Gevrey type satisfied by the initial datum keeps on being satisfied at least for a finite time by the solution. We shall come back to this result later.

Note finally that many papers address the important question of the propagation of the behavior of the solution with respect to large $v$ (that is, propagation of moments, evolution of Maxwellian tails, etc.). We do not investigate in this direction in this work.

The plan of the paper is the following:

- in Section 2, we shall present the result in a simpler form and for the socalled Kac model (which is 1-dimensional and describes radially symmetric solutions of the Boltzmann equation);

- in Section 3, we shall generalize the result both to the Boltzmann equation and to more general bounds on the initial datum;

- finally, in Section 4, we shall recall the main definitions of Gevrey functions, and we shall state the propagation result of a Gevrey-type regularity. 


\section{The Kac equation}

In this section, we present our result in the simpler case of the Kac equation. This equation, in its cut-off or non-cut-off version, is obtained when one considers radially symmetric solutions of the homogeneous Boltzmann equation for Maxwellian molecules. It reads:

$$
\begin{aligned}
\partial_{t} f(v, t) & =\int_{w \in \mathbb{R}} \int_{\theta \in\left[-\frac{\pi}{2}, \frac{\pi}{2}\right]}\left(f\left(\tilde{v}_{*}, t\right) f\left(\tilde{w}_{*}, t\right)-f(v, t) f(w, t)\right) b(\theta) \mathrm{d} \theta \mathrm{d} w \\
& =\tilde{Q}(f, f)(v, t) .
\end{aligned}
$$

Here, $f(v, t): \mathbb{R} \times \mathbb{R}^{+} \longrightarrow \mathbb{R}$ is the probability density of a gas of one dimensional particles which depends only on the velocity $v \in \mathbb{R}$ at the time $t \geq 0$, and which evolves through collisions which conserve energy but not momentum. The relations between the velocities $\left(\tilde{v}_{*}, \tilde{w}_{*}\right)$ of two particles before the collision and $(v, w)$ after it are the following:

$$
\left\{\begin{array}{l}
\tilde{v}_{*}=v \cos \theta+w \sin \theta \\
\tilde{w}_{*}=-v \sin \theta+w \cos \theta .
\end{array}\right.
$$

We shall make the following assumption on the collision kernel $b$ : the function $b$ is even, locally integrable on $\left.] 0, \frac{\pi}{2}\right]$, and such that

$$
\left.b(\theta)=O_{\theta \rightarrow 0}\left(\frac{\cos \theta}{|\sin \theta|^{\gamma}}\right), \quad \text { for some } \quad \gamma \in\right] 1,3[.
$$

Actually, this kind of assumption for the Kac equation was introduced by Desvillettes in [5] whereas, in the original equation, $b(\theta)$ is a strictly positive constant. In the same way as for the Boltzmann equation, it is useful to consider the Cauchy problem in the Fourier variable

$$
\left\{\begin{aligned}
\partial_{t} \hat{f}(\xi, t) & =\int_{\theta \in\left[-\frac{\pi}{2}, \frac{\pi}{2}\right]}(\hat{f}(\xi \cos \theta, t) \hat{f}(\xi \sin \theta, t)-\hat{f}(\xi, t) \hat{f}(0, t)) b(\theta) \mathrm{d} \theta \\
& =\widehat{Q}(f, f)(\xi, t) \\
\hat{f}(\xi, 0)= & \hat{f}_{0}(\xi)
\end{aligned}\right.
$$

where the even initial datum $f_{0} \geq 0$ satisfies the assumptions:

$$
\int_{\mathbb{R}} f_{0}(v) \mathrm{d} v=1, \quad \int_{\mathbb{R}} v^{2} f_{0}(v) \mathrm{d} v=1, \quad \int_{\mathbb{R}} f_{0}(v)\left|\log f_{0}(v)\right| \mathrm{d} v<\infty .
$$

The Kac equation shares with the homogeneous Boltzmann equation for Maxwellian molecules the existence and uniqueness theory for the solutions. By considering the sequence of cut-off approximating problems

$$
\left\{\begin{array}{l}
\partial_{\tau} \varphi_{l}(\xi, \tau)=\int_{\theta \in\left[-\frac{\pi}{2}, \frac{\pi}{2}\right]}\left(\varphi_{l}(\xi \cos \theta, \tau) \varphi_{l}(\xi \sin \theta, \tau)-\varphi_{l}(\xi, \tau) \varphi_{l}(0, \tau)\right) \beta_{l}(\theta) \mathrm{d} \theta, \\
\varphi_{l}(\xi, 0)=\hat{f}_{0}(\xi),
\end{array}\right.
$$

where each $\beta_{l}(\theta)$ is a bounded function defined as in (1.4), (1.5) and (1.6), it is possible to prove that each Cauchy problem (2.4) has a unique solution $\varphi_{l}$, which has the following explicit representation, called Wild's expansion:

$$
\varphi_{l}(\xi, \tau)=\mathrm{e}^{-\tau} \sum_{n=0}^{\infty} \varphi_{l}^{(n)}(\xi)\left(1-\mathrm{e}^{-\tau}\right)^{n},
$$


where

$$
\begin{aligned}
& \varphi_{l}^{(0)}(\xi)=\hat{f}_{0}(\xi) \\
& \varphi_{l}^{(n+1)}(\xi)=\frac{1}{n+1} \sum_{j=0}^{n} \int_{\theta \in\left[-\frac{\pi}{2}, \frac{\pi}{2}\right]} \varphi_{l}^{(j)}(\xi \cos \theta) \varphi_{l}^{(n-j)}(\xi \sin \theta) \beta_{l}(\theta) \mathrm{d} \theta
\end{aligned}
$$

Finally, letting

$$
\hat{f}_{l}(\xi, t):=\varphi_{l}\left(\xi, b_{l}^{*} t\right)
$$

it is possible to establish the (uniform on compact sets) convergence of a subsequence of $\hat{f}_{l}$ to a solution $\hat{f}$ of the Cauchy problem for the (not necessarily cut-off) equation (2.2).

Let us suppose now that the initial datum $f_{0}$ satisfies the extra property:

$$
\left|\hat{f}_{0}(\xi)\right| \leq \mathrm{e}^{-K|\xi|^{s}}, \quad \xi \in \mathbb{R}, K>0, s \in(0,2]
$$

Thanks to the representation of the solution of the cut-off equation (2.4) in Wild's expansion, it is straightforward to prove that the solution itself satisfies the same upper bound. Indeed, by a direct computation, we have that $\left|\varphi_{l}^{(1)}(\xi)\right| \leq \mathrm{e}^{-K|\xi|^{s}}$, since

$$
\begin{aligned}
& \varphi_{l}^{(1)}(\xi) \mathrm{e}^{K|\xi|^{s}} \\
= & \int_{\theta} \mathrm{e}^{K|\xi|^{s}-K|\xi \cos \theta|^{s}-K|\xi \sin \theta|^{s}} \hat{f}_{0}(\xi \cos \theta) \mathrm{e}^{K|\xi \cos \theta|^{s}} \hat{f}_{0}(\xi \sin \theta) \mathrm{e}^{K|\xi \sin \theta|^{s}} \beta_{l}(\theta) \mathrm{d} \theta,
\end{aligned}
$$

and $1-|\cos \theta|^{s}-|\sin \theta|^{s} \leq 0$, for $s \in(0,2]$. Hence by an immediate iteration argument, the same inequality holds for any $\varphi_{l}^{(n)}(\xi)$, and finally for the solution of the cut-off equation for any $t \geq 0$. Passing to the limit when $l \rightarrow+\infty$ in the estimate $\varphi_{l}\left(\xi, b_{l}^{*} t\right) \leq \mathrm{e}^{-K|\xi|^{s}}$, we see that the inequality also holds for the solution of the (not necessarily cut-off) equation (2.2).

Due to the nonlinearity of the collision operator, if we now consider the weaker assumption

$$
\left|\hat{f}_{0}(\xi)\right| \leq K_{1} \mathrm{e}^{-K_{2}|\xi|^{s}}, \quad \xi \in \mathbb{R}, K_{1}>1, K_{2}>0, s \in(0,2],
$$

the same argument allows us to prove that the solution of each cut-off equation (2.4) satisfies the same upper bound, but only for a finite interval of time. In this case, by letting $l$ go to infinity, the interval of time where the estimate is true can reduce to nothing.

In spite of this, we prove in this section that the condition $\left|\hat{f}_{0}(\xi)\right| \leq K_{1} \mathrm{e}^{-K_{2}|\xi|^{s}}$ propagates (though possibly with different constants $K_{1}$ and $K_{2}$ ) along the solution of the (not necessarily cut-off) Kac equation.

2.1. Some preliminary properties of initial data. In this section we emphasize some useful properties satisfied by any even, nonnegative function $g$ such that $\int_{\mathbb{R}} g(v) \mathrm{d} v=1$ and $\int_{\mathbb{R}} v^{2} g(v) \mathrm{d} v=1$.

Lemma 2.1. Let $g$ be a nonnegative, even function, satisfying

$$
\int_{\mathbb{R}} g(v) \mathrm{d} v=1, \quad \int_{\mathbb{R}} g(v) v^{2} \mathrm{~d} v=1 .
$$

Then, there exist $\rho>0$ and $\tilde{K}>0$ such that for all $|\xi| \leq \rho$ :

$$
|\hat{g}(\xi)| \leq \mathrm{e}^{-\tilde{K}|\xi|^{2}}
$$


Proof. We observe that under the hypotheses of the lemma, $\hat{g}$ is of class $C^{2}$ and satisfies the following property: $\hat{g}(0)=1, \hat{g}^{\prime}(0)=0$, and $\hat{g}^{\prime \prime}(0)=-1$. Using a Taylor expansion of $\hat{g}$ at order 2 , we obtain $\hat{g}(\xi)=1-\frac{1}{2} \xi^{2}+o\left(\xi^{2}\right)$ when $\xi \rightarrow 0$. Then, the estimate of the lemma holds for any $\tilde{K} \in] 0, \frac{1}{2}[$.

Then, we prove the following lemma, which is a simplified, one-dimensional version of Lemma 3 in [1].

Lemma 2.2. Let $g \geq 0$ such that $\int_{\mathbb{R}} g(v) \mathrm{d} v=1$. Then, for all $r>0$, there exist $C_{r} \in\left(0, \frac{1}{2}\right)$ and $\tilde{C}_{r} \in\left(0, \frac{1}{2}\right)$ such that

$$
\begin{array}{ll}
\int_{\mathbb{R}} g(v) \sin ^{2}\left(\frac{v \xi}{2}\right) \mathrm{d} v \geq C_{r}, & |\xi|>r, \\
\int_{\mathbb{R}} g(v) \cos ^{2}\left(\frac{v \xi}{2}\right) \mathrm{d} v \geq \tilde{C}_{r}, & |\xi|>r .
\end{array}
$$

Proof. We only prove the first inequality, since the second one can be proven in exactly the same way.

Thanks to Lebesgue's dominated convergence theorem and thanks to the absolute continuity of the measure $\nu(E):=\int_{E} g(v) \mathrm{d} v$ with respect to the Lebesgue measure, there exist $R>0$ and $\delta>0$ such that for all measurable sets $A \subset \mathbb{R}$ such that $|A| \leq \delta$, we have

$$
\int_{A^{c} \cap B(0, R)} g(v) \mathrm{d} v \geq \frac{1}{2} .
$$

Let $\xi \in \mathbb{R}$ be fixed. For $\mu \in] 0, \pi / 2[$, we define

$$
K_{\mu, R}:=\left\{v \in \mathbb{R},|v| \leq R \text { and } \exists k \in \mathbb{Z},\left|\frac{v \xi}{2}-k \pi\right| \leq \mu\right\} .
$$

It is clear that

$$
\left|K_{\mu, R}\right| \leq\left(\frac{|\xi| R}{\pi}+1\right) \frac{4 \mu}{|\xi|}=4 \mu\left(\frac{R}{\pi}+\frac{1}{|\xi|}\right)
$$

so that, when $|\xi| \geq r$, we have $\left|K_{\mu, R}\right| \leq 4 \mu\left(\frac{R}{\pi}+\frac{1}{r}\right)$. When $\mu=\frac{\delta}{4\left(\frac{R}{\pi}+\frac{1}{r}\right)}$, we see thanks to (2.5) that

We can therefore conclude that

$$
\int_{K_{\mu, R}^{c} \cap B(0, R)} g(v) \mathrm{d} v \geq \frac{1}{2} .
$$

$$
\int_{\mathbb{R}} g(v) \sin ^{2}\left(\frac{v \xi}{2}\right) \mathrm{d} v \geq \int_{K_{\mu, R}^{c} \cap B(0, R)} g(v) \sin ^{2}\left(\frac{v \xi}{2}\right) \mathrm{d} v \geq \frac{1}{2} \sin ^{2} \mu:=C_{r} .
$$

2.2. The propagation theorem. We are now in position to state the theorem.

Theorem 2.3. Let $f_{0}$ be a nonnegative, even function, satisfying

$$
\int_{\mathbb{R}} f_{0}(v) \mathrm{d} v=1, \quad \int_{\mathbb{R}} f_{0}(v) v^{2} \mathrm{~d} v=1, \quad \int_{\mathbb{R}} f_{0}(v)\left|\log f_{0}(v)\right| \mathrm{d} v<\infty .
$$

We suppose that $f_{0}$ is such that

$$
\sup _{\xi \in \mathbb{R}}\left|\hat{f}_{0}(\xi)\right| \mathrm{e}^{K_{2}|\xi|^{s}} \leq K_{1}
$$


for some $K_{1} \geq 1, K_{2}>0$, and $0<s \leq 2$. Then there exist $R_{0}>0, K>0$ such that the unique solution of the Cauchy problem (2.2) (under condition (2.1)) satisfies:

$$
\begin{aligned}
& \sup _{|\xi|<R_{0}}|\hat{f}(\xi, t)| \mathrm{e}^{K|\xi|^{2}} \leq 1, \quad t \geq 0 \\
& \sup _{|\xi| \geq R_{0}}|\hat{f}(\xi, t)| \mathrm{e}^{K|\xi|^{s}} \leq 1, \quad t \geq 0 .
\end{aligned}
$$

We begin by proving the following proposition.

Proposition 2.4. Let $g$ be a nonnegative, even function, satisfying

$$
\int_{\mathbb{R}} g(v) \mathrm{d} v=1, \quad \int_{\mathbb{R}} g(v) v^{2} \mathrm{~d} v=1 .
$$

Let us suppose moreover that, for given $s \in(0,2], K_{1}>1$ and $K_{2}>0, g$ satisfies the following bound:

$$
|\hat{g}(\xi)| \leq K_{1} \mathrm{e}^{-K_{2}|\xi|^{s}}, \quad \xi \in \mathbb{R} .
$$

Then, there exists $\eta>0$ such that for all $R>\eta$, there exists $K>0$ (depending on R) such that

$$
|\hat{g}(\xi)| \leq \begin{cases}\mathrm{e}^{-K|\xi|^{2}}, & |\xi|<R \\ \mathrm{e}^{-K|\xi|^{s}}, & |\xi| \geq R\end{cases}
$$

Proof. We have already proven in Lemma 2.1 that $|\hat{g}(\xi)| \leq \mathrm{e}^{-\tilde{K}|\xi|^{2}}$ for $|\xi| \leq \rho$, where $\tilde{K}$ and $\rho$ are suitably chosen. Now, let $\eta=\left(\frac{\log K_{1}}{K_{2}}\right)^{\frac{1}{s}}$. For every $R>\eta$, we can find $0<K_{3}<K_{2}$ such that

$$
K_{1} \mathrm{e}^{-K_{2}|\xi|^{s}} \leq \mathrm{e}^{-K_{3}|\xi|^{s}}, \quad|\xi| \geq R
$$

so that

$$
|\hat{g}(\xi)| \leq \mathrm{e}^{-K_{3}|\xi|^{s}}, \quad|\xi| \geq R .
$$

It is now enough to find $K_{4}>0$ such that

$$
|\hat{g}(\xi)| \leq \mathrm{e}^{-K_{4}|\xi|^{2}}, \quad \rho<|\xi|<R .
$$

Since $g$ is an even function, we have

$$
\hat{g}(\xi)=\int_{\mathbb{R}} g(v) \mathrm{e}^{-i \xi v} \mathrm{~d} v=\int_{\mathbb{R}} g(v)\left(\frac{\mathrm{e}^{-i \xi v}+\mathrm{e}^{i \xi v}}{2}\right) \mathrm{d} v=\int_{\mathbb{R}} g(v) \cos (\xi v) \mathrm{d} v .
$$

Then, $\hat{g}$ is real and $|\hat{g}(\xi)| \leq 1$ for all $\xi \in \mathbb{R}$. Moreover

$$
\begin{aligned}
& 1-\hat{g}(\xi)=\int_{\mathbb{R}} g(v)(1-\cos (\xi v)) \mathrm{d} v=2 \int_{\mathbb{R}} g(v) \sin ^{2}\left(\frac{\xi v}{2}\right) \mathrm{d} v, \\
& \hat{g}(\xi)+1=\int_{\mathbb{R}} g(v)(1+\cos (\xi v)) \mathrm{d} v=2 \int_{\mathbb{R}} g(v) \cos ^{2}\left(\frac{\xi v}{2}\right) \mathrm{d} v .
\end{aligned}
$$

According to Lemma 2.2, we know that

$$
\begin{array}{ll}
1-\hat{g}(\xi) \geq 2 C_{\rho}, & |\xi|>\rho, \\
\hat{g}(\xi)+1 \geq 2 \tilde{C}_{\rho}, & |\xi|>\rho .
\end{array}
$$

Therefore,

$$
|\hat{g}(\xi)| \leq 1-\min \left(2 C_{\rho}, 2 \tilde{C}_{\rho}\right), \quad|\xi|>\rho .
$$


Now, there exists $K_{4}>0$ such that

$$
1-\min \left(2 C_{\rho}, 2 \tilde{C}_{\rho}\right) \leq \mathrm{e}^{-K_{4} R^{2}}
$$

which implies

$$
|\hat{g}(\xi)| \leq 1-\min \left(2 C_{\rho}, 2 \tilde{C}_{\rho}\right) \leq \mathrm{e}^{-K_{4} R^{2}} \leq \mathrm{e}^{-K_{4}|\xi|^{2}}, \quad \rho<|\xi| \leq R .
$$

We can conclude the proof by letting $K=\min \left(\tilde{K}, K_{3}, K_{4}\right)$.

Proof of Theorem 2.3. The cut-off case. There exists $\eta>0$ such that for any $R_{0}>$ $\eta$, there exists a strictly positive $K$ such that the initial datum $\hat{f}_{0}$ satisfies

$$
\begin{aligned}
& \sup _{|\xi|<R_{0}}\left|\hat{f}_{0}(\xi)\right| \mathrm{e}^{K|\xi|^{2}} \leq 1, \\
& \sup _{|\xi| \geq R_{0}}\left|\hat{f}_{0}(\xi)\right| \mathrm{e}^{K|\xi|^{s}} \leq 1 .
\end{aligned}
$$

In order to prove the theorem for the cut-off case, it is enough to establish that any $\varphi_{l}^{(n)}$ in Wild's sums satisfies (2.7). Let us check that this is true for $\varphi_{l}^{(1)}$. Let us define

$$
H(|\xi|)= \begin{cases}K|\xi|^{2}, & |\xi|<R_{0}, \\ K|\xi|^{s}, & |\xi| \geq R_{0} .\end{cases}
$$

Condition (2.7) on the initial datum $f_{0}$ reads therefore

$$
\sup _{\xi \in \mathbb{R}}\left|\hat{f}_{0}(\xi)\right| \mathrm{e}^{H(|\xi|)} \leq 1 .
$$

Then

$$
\begin{aligned}
\left|\mathrm{e}^{H(|\xi|)} \varphi_{l}^{(1)}(\xi)\right| & \leq \int_{\theta \in\left[-\frac{\pi}{2}, \frac{\pi}{2}\right]} \mathrm{e}^{H(|\xi|)-H(|\xi \sin \theta|)-H(|\xi \cos \theta|)} \\
& \leq \int_{\theta \in\left[-\frac{\pi}{2}, \frac{\pi}{2}\right]} \mathrm{e}^{H(|\xi|)-H(|\xi \sin \theta|)-H(|\xi \cos \theta|)} \beta_{l}(\theta) \mathrm{d} \theta .
\end{aligned}
$$

Since $\int_{\theta} \beta_{l}(\theta) \mathrm{d} \theta=1$, we end the estimate by proving that $H(|\xi|)-H(|\xi \sin \theta|)-$ $H(|\xi \cos \theta|) \leq 0$ for $\xi \in \mathbb{R}$ and $\theta \in\left[-\frac{\pi}{2}, \frac{\pi}{2}\right]$ if $R_{0} \geq 1$. Thanks to the symmetries of the function $H$ with respect to $\theta$, we can restrict ourselves to the interval $\left[0, \frac{\pi}{4}\right]$. Now, when $|\xi|<R_{0}$, we have

$$
H(|\xi|)-H(|\xi \sin \theta|)-H(|\xi \cos \theta|)=K|\xi|^{2}\left(1-(\sin \theta)^{2}-(\cos \theta)^{2}\right)=0 .
$$

If $|\xi| \geq R_{0}$ and $|\xi \sin \theta| \geq R_{0},|\xi \cos \theta| \geq R_{0}$, then

$$
H(|\xi|)-H(|\xi \sin \theta|)-H(|\xi \cos \theta|)=K|\xi|^{s}\left(1-(\sin \theta)^{s}-(\cos \theta)^{s}\right) \leq 0
$$

for $0<s \leq 2$. Whenever $|\xi| \geq R_{0}$ and $|\xi \sin \theta|<R_{0},|\xi \cos \theta|<R_{0}$ we have

$$
\begin{aligned}
H(|\xi|)-H(|\xi \sin \theta|)-H(|\xi \cos \theta|) & =K\left(|\xi|^{s}-|\xi|^{2}\left((\sin \theta)^{2}+(\cos \theta)^{2}\right)\right) \\
& =K\left(|\xi|^{s}-|\xi|^{2}\right) .
\end{aligned}
$$

If we choose $R_{0} \geq 1$, we can conclude, since $|\xi| \geq R_{0}$, that

$$
|\xi|^{s}-|\xi|^{2} \leq 0 \text {. }
$$


If now $|\xi| \geq R_{0}$ and $|\xi \sin \theta|<R_{0},|\xi \cos \theta| \geq R_{0}$, we have

$$
\begin{gathered}
H(|\xi|)-H(|\xi \sin \theta|)-H(|\xi \cos \theta|)=K\left(|\xi|^{s}-|\xi|^{2}(\sin \theta)^{2}-|\xi|^{s}(\cos \theta)^{s}\right) \\
\leq K\left(|\xi|^{s}-|\xi|^{s}(\sin \theta)^{2}-|\xi|^{s}(\cos \theta)^{2}\right)=0 .
\end{gathered}
$$

Note that, since $0 \leq \theta \leq \pi / 4$, there is no other case to treat.

The non-cut-off case. As we have recalled in the introduction of section 2 , the solution $\hat{f}(\xi, t)$ of (2.2) in the non-cut-off case is obtained as the limit, uniform on compact sets, of a subsequence of $\varphi_{l}\left(\xi, b_{l}^{*} t\right)$, where $\varphi_{l}(\xi, \tau)$ is the solution of the Cauchy problem (2.4). Here $b_{l}^{*}=\int_{\theta} \min (b(\theta), l) \mathrm{d} \theta$. Since the estimate on $\varphi_{l}(\xi, \tau)$ holds true uniformly for $\tau \geq 0$, the same is valid for $\varphi_{l}\left(\xi, b_{l}^{*} t\right)$ and hence for $\hat{f}(\xi, t)$.

Remark 2.5. In Theorem 2.3, the hypothesis that $f_{0}$ is even could be replaced by the weaker hypothesis that $\int_{\mathbb{R}} f_{0}(v) v \mathrm{~d} v=0$. Since the Kac equation comes from the Boltzmann equation when one considers radially symmetric solutions, it is however natural to study only even initial data.

\section{Propagation for the Boltzmann equation}

We would like to extend to the solution of the Boltzmann equation (1.3) the results proven in the previous section for the solution of the Kac equation. Two kinds of extensions are in order: first, we have to pass from the one-dimensional to the three-dimensional setting; second we would like to state the result considering not only functions such as $\mathrm{e}^{-|\xi|^{s}}$, but also such as $\mathrm{e}^{-\psi\left(|\xi|^{2}\right)}$, where $\psi$ is a suitable function. We now begin by restating the lemmas of the previous section in three dimensions. We shall only indicate the major modifications in the proofs.

Lemma 3.1. Let $g: \mathbb{R}^{3} \rightarrow \mathbb{R}$ be a nonnegative function satisfying

$$
\int_{\mathbb{R}^{3}} g(v) \mathrm{d} v=1, \quad \int_{\mathbb{R}^{3}} g(v) v_{i} \mathrm{~d} v=0, i=1,2,3, \quad \int_{\mathbb{R}^{3}} g(v)|v|^{2} \mathrm{~d} v=3 .
$$

Then there exist $\rho>0$ and $\tilde{K}>0$ such that for all $|\xi| \leq \rho$ :

$$
|\hat{g}(\xi)| \leq \mathrm{e}^{-\tilde{K}|\xi|^{2}} \text {. }
$$

Proof. We observe that the result of the lemma is not changed when $g$ is replaced by $g \circ R$, where $R$ is any rotation of $\mathbb{R}^{3}$. As a consequence, we can suppose that the symmetric matrix $\left(\int_{\mathbb{R}^{3}} g(v) v_{i} v_{j} \mathrm{~d} v\right)_{i, j \in\{1,2,3\}}$ is diagonal. Moreover, since $g \in$ $L^{1}\left(\mathbb{R}^{3}\right)$ and $\int_{v \in \mathbb{R}^{3}} g(v)|v|^{2} \mathrm{~d} v=3$, we see that $\int_{v \in \mathbb{R}^{3}} g(v) v_{i}^{2} \mathrm{~d} v>0$ for $i=1,2,3$.

Then, $\hat{g}(\xi)=1-\sum_{j=1}^{3} \lambda_{j} \xi_{j}^{2}+o_{\xi \rightarrow 0}\left(|\xi|^{2}\right)$, with $\lambda_{j}>0$ for $j=1,2,3$, and we conclude the proof as in Lemma 2.1

Lemma 3.2. Let $g: \mathbb{R}^{3} \rightarrow \mathbb{R}$ be a nonnegative function satisfying $\int_{\mathbb{R}^{3}} g(v) \mathrm{d} v=1$. Then, for all $r>0$, there exists $C_{r} \in\left(0, \frac{1}{2}\right)$ such that for all $\theta \in \mathbb{R}$,

$$
\int_{\mathbb{R}^{3}} g(v) \sin ^{2}\left(\frac{v \cdot \xi+\theta}{2}\right) \mathrm{d} v \geq C_{r}, \quad|\xi|>r .
$$

Proof. The proof follows the same lines as that of Lemma 2.2 and is a simplified version of Lemma 3 in [1]. Let $\xi \in \mathbb{R}^{3}$ be fixed. We start by choosing in $\mathbb{R}^{3}$ an orthogonal system in which the unitary vector along the $z$-axis is $\frac{\xi}{|\xi|}$. As in the 
one-dimensional case, there exist $R>0$ and $\delta>0$ such that for all measurable sets $A \subset \mathbb{R}^{3}$ such that $|A|<\delta$, we have

$$
\int_{A^{c} \cap Q(0, R)} g(v) \mathrm{d} v \geq \frac{1}{2},
$$

where $Q(0, R)$ is the cube centered at the origin:

$$
Q(0, R)=\left\{v \in \mathbb{R}^{3}:\left|v_{i}\right| \leq R, i=1,2,3\right\} .
$$

Now, for $\mu \in] 0, \frac{\pi}{2}[$, we define

$$
K_{\mu, R, \theta}:=\left\{v \in \mathbb{R}^{3}, v \in Q(0, R) \text { and } \exists k \in \mathbb{Z},\left|\frac{v \cdot \xi+\theta}{2}-k \pi\right| \leq \mu\right\} .
$$

Thanks to the choice of the coordinate system, we have

$$
K_{\mu, R, \theta}=\left\{v \in \mathbb{R}^{3},\left|v_{i}\right| \leq R, i=1,2,3 \text { and } \exists k \in \mathbb{Z},\left|\frac{v_{3}|\xi|+\theta}{2}-k \pi\right| \leq \mu\right\} .
$$

So, it is easy to see that

$$
\left|K_{\mu, R, \theta}\right| \leq\left(\frac{|\xi| R}{\pi}+1\right) \frac{4 \mu}{|\xi|} R^{2}=4 \mu R^{2}\left(\frac{R}{\pi}+\frac{1}{|\xi|}\right)
$$

and we can conclude the proof as in the one-dimensional case.

We are now in a position to prove the main theorem of our paper, namely Theorem 1.2. The proof of this theorem relies on the following proposition:

Proposition 3.3. Let $g$ be a nonnegative function satisfying

$$
\int_{\mathbb{R}^{3}} g(v) \mathrm{d} v=1, \quad \int_{\mathbb{R}^{3}} g(v) v_{i} \mathrm{~d} v=0, i=1,2,3, \quad \int_{\mathbb{R}^{3}} g(v)|v|^{2} \mathrm{~d} v=3 .
$$

Let us suppose moreover that

$$
|\hat{g}(\xi)| \leq K_{1} \mathrm{e}^{-K_{2} \varphi(|\xi|)}, \quad \xi \in \mathbb{R}^{3},
$$

where $K_{1} \geq 1, K_{2}>0$ and $\varphi:[0,+\infty) \rightarrow[0,+\infty)$ satisfies $\lim _{t \rightarrow+\infty} \varphi(t)=+\infty$. Then, there exists $\eta>0$ such that for all $R>\eta$, there exists $K>0$ (depending on R) such that

$$
|\hat{g}(\xi)| \leq \begin{cases}\mathrm{e}^{-K|\xi|^{2}}, & |\xi|<R \\ \mathrm{e}^{-K \varphi(|\xi|)}, & |\xi| \geq R .\end{cases}
$$

Proof. The proof of this proposition is only a slight modification of that of Proposition 2.4. We only point out the few differences.

First, we explain how to find $\eta>0$ : we can fix $K_{3} \in\left(0, K_{2}\right)$ and let $\eta=\eta\left(K_{3}\right)$ be a positive constant such that $\varphi(|\xi|) \geq \frac{1}{K_{2}-K_{3}} \log K_{1}$ for every $|\xi| \geq \eta$; then of course for every $R \geq \eta$,

$$
K_{1} \mathrm{e}^{-K_{2} \varphi(|\xi|)} \leq \mathrm{e}^{-K_{3} \varphi(|\xi|)}, \quad|\xi| \geq R .
$$

Second, we observe that for all $\xi \in \mathbb{R}^{3}$ it is possible to find $\theta \in \mathbb{R}$ (depending on $\xi)$ such that

So, we have

$$
|\hat{g}(\xi)|=\hat{g}(\xi) \mathrm{e}^{i \theta}=\int_{\mathbb{R}^{3}} g(v) \cos (\xi \cdot v+\theta) \mathrm{d} v
$$

$$
1-|\hat{g}(\xi)|=2 \int_{\mathbb{R}^{3}} g(v) \sin ^{2}\left(\frac{\xi \cdot v+\theta}{2}\right) \mathrm{d} v .
$$


Thanks to Lemma 3.2, there exists $C_{\rho}$ such that

$$
2 \int_{\mathbb{R}^{3}} g(v) \sin ^{2}\left(\frac{\xi \cdot v+\theta}{2}\right) \mathrm{d} v \geq 2 C_{\rho}, \quad|\xi|>\rho .
$$

Then, we can conclude as in the proof of Proposition 2.4.

Proof of Theorem 1.2. As we did for the Kac equation, for each of the Cauchy problems (1.7), we write the solution $\varphi_{l}$ under the form of a Wild's expansion.

In order to prove the bound for the solution, it is enough to prove it for every term $\varphi_{l}^{(n)}$ in the sum. We define

$$
H(|\xi|)= \begin{cases}K|\xi|^{2}, & |\xi|<R_{0}, \\ K \psi\left(|\xi|^{2}\right), & |\xi| \geq R_{0},\end{cases}
$$

where $R_{0} \geq \bar{R}$ will be chosen (large enough) later, and $K$ is given by Proposition 3.3. Thanks to this proposition, the initial datum satisfies

$$
\sup _{\xi \in \mathbb{R}^{3}}\left|\hat{f}_{0}(\xi)\right| \mathrm{e}^{H(|\xi|)} \leq 1 .
$$

We recall the identities ([], page 56)

$$
\begin{aligned}
& \left|\xi^{+}\right|=|\xi| \cos \frac{\theta}{2} \\
& \left|\xi^{-}\right|=|\xi| \sin \frac{\theta}{2} .
\end{aligned}
$$

For the first term $\varphi_{l}^{(1)}$ we have:

$$
\begin{aligned}
& \mid \varphi_{l}^{(1)}(\xi) \mathrm{e}^{H(|\xi|) \mid} \\
& =\left|\int_{S^{2}} \mathrm{e}^{H(|\xi|)-H\left(\left|\xi^{+}\right|\right)-H\left(\left|\xi^{-}\right|\right)} \hat{f}_{0}\left(\xi^{+}\right) \mathrm{e}^{H\left(\left|\xi^{+}\right|\right)} \hat{f}_{0}\left(\xi^{-}\right) \mathrm{e}^{H\left(\left|\xi^{-}\right|\right)} \beta_{l}\left(\frac{\xi}{|\xi|} \cdot n\right) \mathrm{d} n\right| \\
& \leq \int_{\theta \in(0, \pi)} \int_{\varphi \in(0,2 \pi)} \mathrm{e}^{H(|\xi|)-H\left(|\xi| \cos \frac{\theta}{2}\right)-H\left(|\xi| \sin \frac{\theta}{2}\right)} \beta_{l}(\cos \theta) \sin \theta \mathrm{d} \theta \mathrm{d} \varphi,
\end{aligned}
$$

where in the last integral, we have used the spherical coordinates with $\frac{\xi}{|\xi|}$ as $z$-axis. Then, in order to establish $\left|\varphi_{l}^{(1)}(\xi) \mathrm{e}^{H(|\xi|)}\right| \leq 1$, we show that for $R_{0}$ large enough,

$$
H(|\xi|)-H\left(\left|\xi \cos \frac{\theta}{2}\right|\right)-H\left(\left|\xi \sin \frac{\theta}{2}\right|\right) \leq 0, \quad \theta \in(0, \pi), \xi \in \mathbb{R}^{3} .
$$

We denote $\tilde{\theta}=\frac{\theta}{2}$. Thanks to the symmetries of the functions $H(|\xi|)-H(|\xi \sin \tilde{\theta}|)-$ $H(|\xi \cos \tilde{\theta}|)$ with respect to $\tilde{\theta}$, we can restrict ourselves to the interval $\left(0, \frac{\pi}{4}\right)$. Now, the case $|\xi|<R_{0}$ is the same as in Theorem 2.3. If $|\xi| \geq R_{0}$ and $|\xi \sin \tilde{\theta}| \geq R_{0}$, $|\xi \cos \tilde{\theta}| \geq R_{0}$, then

$$
H(|\xi|)-H(|\xi \sin \tilde{\theta}|)-H(|\xi \cos \tilde{\theta}|)=K\left(\psi\left(|\xi|^{2}\right)-\psi\left(|\xi \sin \tilde{\theta}|^{2}\right)-\psi\left(|\xi \cos \tilde{\theta}|^{2}\right)\right) .
$$

Thanks to the $(H)$ property of $\psi$ we have $\psi\left(|\xi|^{2}(\sin \tilde{\theta})^{2}\right) \geq(\sin \tilde{\theta})^{2} \psi\left(|\xi|^{2}\right)$ and $\psi\left(|\xi|^{2}(\cos \tilde{\theta})^{2}\right) \geq(\cos \tilde{\theta})^{2} \psi\left(|\xi|^{2}\right)$. Hence

$\psi\left(|\xi|^{2}\right)-\psi\left(|\xi \sin \tilde{\theta}|^{2}\right)-\psi\left(|\xi \cos \tilde{\theta}|^{2}\right) \leq \psi\left(|\xi|^{2}\right)-(\sin \tilde{\theta})^{2} \psi\left(|\xi|^{2}\right)-(\cos \tilde{\theta})^{2} \psi\left(|\xi|^{2}\right)=0$. 
Whenever $|\xi| \geq R_{0}$ and $|\xi \sin \tilde{\theta}|<R_{0},|\xi \cos \tilde{\theta}|<R_{0}$ we have

$$
\begin{aligned}
H(|\xi|)-H(|\xi \sin \tilde{\theta}|)-H(|\xi \cos \tilde{\theta}|) & =K\left(\psi\left(|\xi|^{2}\right)-|\xi|^{2}\left((\sin \tilde{\theta})^{2}+(\cos \tilde{\theta})^{2}\right)\right) \\
& =K\left(\psi\left(|\xi|^{2}\right)-|\xi|^{2}\right) .
\end{aligned}
$$

If we choose $R_{0}$ large enough, thanks to the assumption that $\psi(r) \leq r$ for $r$ large enough, and since $|\xi| \geq R_{0}$, we can conclude that

$$
\psi\left(|\xi|^{2}\right)-|\xi|^{2} \leq 0
$$

By using now the $(H)$ property of $\psi$ and the fact that $\psi(r) \leq r$, if now $|\xi| \geq R_{0}$ and $|\xi \sin \tilde{\theta}|<R_{0},|\xi \cos \tilde{\theta}| \geq R_{0}$ we have

$$
\begin{aligned}
H(|\xi|)-H(|\xi \sin \tilde{\theta}|)-H(|\xi \cos \tilde{\theta}|) & =K\left(\psi\left(|\xi|^{2}\right)-|\xi|^{2}(\sin \tilde{\theta})^{2}-\psi\left(|\xi \cos \tilde{\theta}|^{2}\right)\right) \\
& \leq K\left(\psi\left(|\xi|^{2}\right)-\psi\left(|\xi|^{2}\right)(\sin \tilde{\theta})^{2}-\psi\left(|\xi \cos \tilde{\theta}|^{2}\right)\right) \\
& \leq K \psi\left(|\xi|^{2}\right)\left(1-(\sin \tilde{\theta})^{2}-(\cos \tilde{\theta})^{2}\right)=0
\end{aligned}
$$

We end the proof by first noticing that a simple induction shows the estimate $\left|\varphi_{l}^{(n)}(\xi) \mathrm{e}^{H(|\xi|)}\right| \leq 1$ when $n \geq 1$, and so for the solution $\varphi_{l}(\xi, \tau)$ of the cut-off Cauchy problem, uniformly in $\tau \geq 0$. Then we may pass to the limit when $l \rightarrow+\infty$ for a subsequence of $\varphi_{l}\left(\xi, b_{l}^{*} t\right)$ getting the same result for the solution $\hat{f}(\xi, t)$ of the non-cut-off Cauchy problem.

\section{Gevrey spaces}

In this section, we translate the propagation result obtained in the previous sections in terms of Gevrey regularity for the solutions of the Boltzmann equation. Let us begin by recalling the classical definitions of Gevrey functions and a useful characterisation of these functions through their Fourier transform. For more information, the interested reader can consult for instance the book by Rodino [12, from where we have taken the following recalls.

Let $\Omega \subseteq \mathbb{R}^{n}$ be an open set and let $\nu \geq 1$ be a fixed real number.

Definition 4.1. The class $G^{\nu}(\Omega)$ of Gevrey functions of order $\nu$ in $\Omega$ is the set of functions $f \in C^{\infty}(\Omega)$ satisfying the following property: for every compact subset $K$ of $\Omega$, there exists a positive constant $C=C(K)$ such that for all $l \in \mathbb{N}^{n}$ and all $x \in K$,

$$
\left|\partial^{l} f(x)\right| \leq C^{|l|+1}(l !)^{\nu} .
$$

Assumption (4.1) can be replaced by other equivalent assumptions, for example

$$
\left|\partial^{l} f(x)\right| \leq R C^{|l|}(l !)^{\nu}
$$

where $R$ and $C$ are two positive constants independent of $l$ and $x \in K$. It is easy to recognize that $G^{1}(\Omega)=A(\Omega)$, the space of all analytic functions in $\Omega$, and that for $\nu \leq \tau$, one has $G^{\nu}(\Omega) \subseteq G^{\tau}(\Omega)$. Moreover, it is interesting to underline the following inclusions:

$$
A(\Omega) \subset \bigcap_{\nu>1} G^{\nu}(\Omega), \quad \bigcup_{\nu \geq 1} G^{\nu}(\Omega) \subset C^{\infty}(\Omega),
$$

which are strict in both cases. We also recall that the Gevrey class $G^{\nu}(\Omega)$ is closed under differentiation.

In what follows, we shall also need the following. 
Definition 4.2. Assume $\nu>1$. We shall denote by $G_{0}^{\nu}(\Omega)$ the vector space of all $f \in G^{\nu}(\Omega)$ with compact support in $\Omega$.

The exclusion of $\nu=1$ in the previous definition is mandatory, because there are no analytic test functions other than the zero function. As for the other values $\nu>1$, one could wonder whether such compact supported functions do exist. An example in $\mathbb{R}$ is the following: let $r>0, \nu>1, d=\frac{1}{1-\nu}$ and

$$
\varphi(t)= \begin{cases}\mathrm{e}^{-t^{d}} & t>0 \\ 0 & t \leq 0\end{cases}
$$

The function

$$
f(x)=\varphi(x+r) \varphi(x-r)
$$

is then in $G_{0}^{\nu}(\mathbb{R})([12])$.

The result that we are going to use in order to relate our propagation result to Gevrey regularity is the following.

Theorem 4.3 ([12, Theorem 1.6.1, page 31).

i) Let $\nu>1$. If $\varphi \in G_{0}^{\nu}\left(\mathbb{R}^{n}\right)$, then there exist positive constants $C$ and $\varepsilon$ such that

$$
|\hat{\varphi}(\xi)| \leq C \mathrm{e}^{-\varepsilon|\xi|^{\frac{1}{\nu}}}, \quad \xi \in \mathbb{R}^{n} .
$$

ii) Let $\nu \geq 1$. If the Fourier transform of $\varphi \in \mathcal{S}^{\prime}\left(\mathbb{R}^{n}\right)$ satisfies (4.2), then $\varphi \in G^{\nu}\left(\mathbb{R}^{n}\right)$.

We can therefore deduce Corollary 1.3 concerning the regularity of the solutions of the Boltzmann equation.

Proof of Corollary 1.3, The result is straightforward from Theorem 4.3 and Theorem 1.2. It is enough to notice that one can replace the uniform estimate

$$
\begin{aligned}
& \sup _{|\xi|<R_{0}}|\hat{f}(\xi, t)| \mathrm{e}^{K|\xi|^{2}} \leq 1, \quad t \geq 0, \\
& \sup _{|\xi| \geq R_{0}}|\hat{f}(\xi, t)| \mathrm{e}^{K|\xi|^{s}} \leq 1, \quad t \geq 0,
\end{aligned}
$$

obtained in Theorem 1.2, by the following uniform estimate:

$$
\sup _{\xi \in \mathbb{R}^{3}}|\hat{f}(\xi, t)| \mathrm{e}^{\tilde{K}_{2}|\xi|^{s}} \leq \tilde{K}_{1}, \quad t \geq 0,
$$

for $\tilde{K}_{1} \geq 1$ and $\tilde{K}_{2}>0$ properly chosen. Now letting $\nu=\frac{1}{s}$, one immediately gets the result. This ends the proof.

We end this section by comparing the regularity result we have just obtained with the one obtained by Ukai in 14. In his work, he considered among others a Cauchy problem for the homogeneous Boltzmann equation for Maxwellian molecules both in the cut-off and non-cut-off settings. He considered only initial data $f_{0}$ satisfying a strong regularity assumption: for $\alpha \geq 0, \rho \geq 0$ and $\nu \geq 1$ he supposed $f_{0} \in \gamma_{\alpha, \rho}^{\nu}$, where

$$
\gamma_{\alpha, \rho}^{\nu}=\left\{g:\|g\|_{\alpha, \rho, \nu}=\sum_{l \in \mathbb{N}^{3}} \frac{\rho^{|l|}}{(l !)^{\nu}} \sup _{v \in \mathbb{R}^{3}} \mathrm{e}^{\alpha\left(1+|v|^{2}\right)^{\frac{1}{2}}}\left|\partial^{l} g(v)\right|<\infty\right\} .
$$

Comparing this space with the spaces in Definition 4.1, one can deduce that initial data in the Ukai setting are indeed in the Gevrey space $G^{\nu}\left(\mathbb{R}^{3}\right)$, but also decay very 
strongly at infinity together with all their derivatives. Ukai was able to prove by a fixed point argument that there exists a unique, local in time solution $f$ belonging at every time to a functional space of the same kind as the initial datum but in which the indices change with $t$. More precisely, he proved that there exist $T>0$, $\beta>0, \sigma>0$ such that

$$
\|f(\cdot, t)\|_{\alpha-t \beta, \rho-t \sigma, \nu} \leq 2\left\|f_{0}\right\|_{\alpha, \rho, \nu}, \quad t \in[0, T] .
$$

Since initial data which belong to $G_{0}^{\nu}\left(\mathbb{R}^{3}\right)$ also belong to the Ukai space, the question of comparing the two results is meaningful. Let us consider a nonnegative function $f_{0} \in G_{0}^{\nu}\left(\mathbb{R}^{3}\right)$. If we suppose moreover that $f_{0}$ satisfies assumptions (1.9), then by Theorem 1.1 we know that there is a solution $f \in C^{1}\left([0,+\infty), L^{1}\left(\mathbb{R}^{3}\right)\right)$ which is unique by Toscani-Villani's result. Since $G_{0}^{\nu}\left(\mathbb{R}^{3}\right) \subset \gamma_{\alpha, \rho}^{\nu}$ for all $\alpha \geq 0$, we can deduce from the Ukai result that for all $\alpha \geq 0$ there is a time $T=T(\alpha)>0$ (possibily finite) such that this solution stays in the class (4.3) for $t \in[0, T(\alpha)]$, but this space is not uniform in time (in addition to the fact that for all $\alpha$ it is difficult to compare the life times $T(\alpha)$ ). Our result says instead that the solution stays for $t \in[0, \infty)$ in the same Gevrey class as its initial datum, without any information about the decay at infinity and moreover that all the estimates on the Gevrey seminorms are uniform in time.

\section{ACKNOWLEDGEMENTS}

The authors would like to thank G. Toscani for useful discussions about this problem.

\section{REFERENCES}

1. R. Alexandre, L. Desvillettes, C. Villani, and B. Wennberg. Entropy dissipation and long-range interactions. Arch. Rational Mech. Anal., 152(4):327-355, 2000. MR.1765272 (2001c:82061)

2. L. Arkeryd. Intermolecular forces of infinite range and the Boltzmann equation. Arch. Rational Mech. Anal., 77(1):11-21, 1981. MR630119 (83k:76057)

3. A. V. Bobylëv. The theory of the nonlinear spatially uniform Boltzmann equation for Maxwell molecules. In Mathematical physics reviews, Vol. 7, volume 7 of Soviet Sci. Rev. Sect. C Math. Phys. Rev., pages 111-233. Harwood Academic Publ., Chur, 1988. MR.1128328 (92m:82112)

4. E. A. Carlen, E. Gabetta, and G. Toscani. Propagation of smoothness and the rate of exponential convergence to equilibrium for a spatially homogeneous Maxwellian gas. Comm. Math. Phys., 199(3):521-546, 1999. MR 1669689 (99k:82060)

5. L. Desvillettes. About the regularizing properties of the non-cut-off Kac equation. Comm. Math. Phys., 168(2):417-440, 1995. MR1324404 (96d:82052)

6. L. Desvillettes. About the use of the Fourier transform for the Boltzmann equation. Riv. Mat. Univ. Parma (7), $2 * 1$-99, 2003. Summer School on "Methods and Models of Kinetic Theory" (M\&MKT 2002). MR2052786(2005a:35040)

7. L. Desvillettes and C. Mouhot. About $L^{p}$ estimates for the spatially homogeneous Boltzmann equation. Ann. Inst. H. Poincaré Anal. Non Linéaire, 22(2):127-142, 2005. MR2123118 (2005m:35035)

8. T. Gustafsson. $L^{p}$-estimates for the nonlinear spatially homogeneous Boltzmann equation. Arch. Rational Mech. Anal., 92(1):23-57, 1986. MR816620(87h:82054)

9. T. Gustafsson. Global $L^{p}$-properties for the spatially homogeneous Boltzmann equation. Arch . Rational Mech. Anal., 103(1):1-38, 1988. MR946968 (89h:82019)

10. C. Mouhot and C. Villani. Regularity theory for the spatially homogeneous Boltzmann equation with cut-off. Arch. Rational Mech. Anal., 173(2):169-212, 2004. MR2081030 (2006f:82073)

11. A. Pulvirenti and G. Toscani. The theory of the nonlinear Boltzmann equation for Maxwell molecules in Fourier representation. Ann. Mat. Pura Appl. (4), 171:181-204, 1996. MR:1441870(98m:35212) 
12. L. Rodino. Linear partial differential operators in Gevrey spaces. World Scientific Publishing Co. Inc., River Edge, NJ, 1993. MR.1249275 (95c:35001)

13. G. Toscani and C. Villani. Probability metrics and uniqueness of the solution to the Boltzmann equation for a Maxwell gas. J. Statist. Phys., 94(3-4):619-637, 1999. MR1675367 (2000f:82089)

14. S. Ukai. Local solutions in Gevrey classes to the nonlinear Boltzmann equation without cutoff. Japan J. Appl. Math., 1(1):141-156, 1984. MR839310 (87j:45025)

15. C. Villani. A review of mathematical topics in collisional kinetic theory. In Handbook of mathematical fluid dynamics, Vol. I, pages 71-305. North-Holland, Amsterdam, 2002. MR1942465 $(2003 \mathrm{k}: 82087)$

CMla, ens Cachan, CNRs, Pres UniverSud, 61, Avenue du Président Wilson, 94235 Cachan Cedex, France

E-mail address: desville@cmla.ens-cachan.fr

Dipartimento di Ingegneria dell'Informazione e Metodi Matematici, Università di Bergamo, Viale Marconi 5, I-24044 Dalmine (BG), Italy

E-mail address: gfurioli@unibg.it

Dipartimento di Matematica F. Enriques, Università degli studi di Milano, Via SalDini 50, I-20133 Milano, ItAly

E-mail address: terraneo@mat.unimi.it 